\title{
On the Period-Luminosity-Colour-Metallicity relation and the pulsational characteristics of $\lambda$ Bootis type stars ${ }^{\star}$
}

\author{
E. Paunzen ${ }^{1,2}$, G. Handler ${ }^{3}$, W. W. Weiss ${ }^{1}$, N. Nesvacil ${ }^{1}$, A. Hempel ${ }^{4,5}$, E. Romero-Colmenero ${ }^{3}$, F. F. Vuthela ${ }^{3,6}$, \\ P. Reegen ${ }^{1}$, R. R. Shobbrook ${ }^{7}$, and D. Kilkenny ${ }^{3}$ \\ 1 Institut für Astronomie der Universität Wien, Türkenschanzstr. 17, 1180 Wien, Austria \\ 2 Zentraler Informatikdienst der Universität Wien, Universitätsstr. 7, 1010 Wien, Austria \\ 3 South African Astronomical Observatory, PO Box 9, Observatory 7935, South Africa \\ ${ }^{4}$ Department of Physics, University of Cape Town, Private Bag, Rondebosch 7701, South Africa \\ 5 Max-Planck-Institut für Astronomie, Königsstuhl 17, 69117 Heidelberg, Germany \\ ${ }^{6}$ Department of Physics, University of the North-West, Private Bag X2046, Mmabatho 2735, South Africa \\ 7 Research School of Astronomy and Astrophysics, Australian National University, Canberra, ACT 0200, Australia
}

Received 12 April 2002 / Accepted 6 June 2002

\begin{abstract}
Generally, chemical peculiarity found for stars on the upper main sequence excludes $\delta$ Scuti type pulsation (e.g. Ap and Am stars), but for the group of $\lambda$ Bootis stars it is just the opposite. This makes them very interesting for asteroseismological investigations. The group of $\lambda$ Bootis type stars comprises late B- to early F-type, Population I objects which are basically metal weak, in particular the Fe group elements, but with the clear exception of $\mathrm{C}, \mathrm{N}, \mathrm{O}$ and $\mathrm{S}$. The present work is a continuation of the studies by Paunzen et al. (1997, 1998), who presented first results on the pulsational characteristics of the $\lambda$ Bootis stars. Since then, we have observed 22 additional objects; we found eight new pulsators and confirmed another one. Furthermore, new spectroscopic data (Paunzen 2001) allowed us to sort out misidentified candidates and to add true members to the group. From 67 members of this group, only two are not photometrically investigated yet which makes our analysis highly representative. We have compared our results on the pulsational behaviour of the $\lambda$ Bootis stars with those of a sample of $\delta$ Scuti type objects. We find that at least $70 \%$ of all $\lambda$ Bootis type stars inside the classical instability strip pulsate, and they do so with high overtone modes $(Q<0.020 \mathrm{~d})$. Only a few stars, if any, pulsate in the fundamental mode. Our photometric results are in excellent agreement with the spectroscopic work on high-degree nonradial pulsations by Bohlender et al. (1999). Compared to the $\delta$ Scuti stars, the cool and hot borders of the instability strip of the $\lambda$ Bootis stars are shifted by about 25 mmag, towards smaller $(b-y)_{0}$. Using published abundances and the metallicity sensitive indices of the Geneva 7-colour and Strömgren $u v b y \beta$ systems, we have derived $[Z]$ values which describe the surface abundance of the heavier elements for the group members. We find that the PeriodLuminosity-Colour relation for the group of $\lambda$ Bootis stars is within the errors identical with that of the normal $\delta$ Scuti stars. No clear evidence for a statistically significant metallicity term was detected.
\end{abstract}

Key words. stars $-\lambda$ Bootis ; stars - chemically peculiar; stars - early type

\section{Introduction}

In this paper we present an extensive survey to analyse the pulsational characteristics of the $\lambda$ Bootis stars. This small group comprises late B- to early F-type, Population I stars which are metal weak (particularly the Fe group elements), but with the clear exception of C, N, O and S. Only a maximum of about $2 \%$ of all objects in the relevant spectral domain are believed to be $\lambda$ Bootis type stars.

Send offprint requests to: E. Paunzen, e-mail: ernst.paunzen@univie.ac.at

* Based on observations from the Austrian Automatic Photoelectric Telescope (Fairborn Observatory), SAAO and Siding Spring Observatory.
Several theories were developed to explain the peculiar abundance pattern for members of this group. The most acknowledged models include diffusion as main mechanism together either with mass-loss (Michaud \& Charland 1986; Charbonneau 1993) or with accretion of circumstellar material (Venn \& Lambert 1990; Turcotte \& Charbonneau 1993). Another two theories deal with the influence of binarity on this phenomenon (Andrievsky 1997; Faraggiana \& Bonifacio 1999). Heiter (2002) and Heiter et al. (2002) also tried to explain the abundance pattern in the context of the proposed theories.

In general, chemical peculiarity inhibits $\delta$ Scuti type pulsation (e.g. for Ap and Am stars, see Kurtz 2000 for a recent discussion) but for the group of $\lambda$ Bootis stars it is just the 
opposite. In two previous studies (Paunzen et al. 1997, 1998), we presented non-variable as well as pulsating $\lambda$ Bootis stars. Since then, we have observed 22 additional objects and found eight new pulsators and confirmed another. Furthermore, new spectroscopic data (Paunzen 2001) has allowed us to sort out misidentified candidates and to add true members of the group.

Turcotte et al. (2000) investigated the effect of diffusion (probably the main cause of the $\lambda$ Bootis phenomenon) on the pulsation of stars at the upper main sequence. Although these authors mainly investigated the theoretical behaviour of apparently metal-rich objects, their conclusions also have an impact for the $\lambda$ Bootis group: little direct pulsational excitation from Fe-peak elements was found, but effects due to settling of helium along with the enhancement of hydrogen are important. Turcotte et al. (2000) find that, as their models of peculiar stars evolve, they become generally pulsationally unstable near the red edge of the instability strip, whereas the behaviour at the blue edge is mainly sensitive to the surface metal abundance. Although the proposed models are still simplified (e.g. treatment of convection) these preliminary results already point towards the most important effects on the theoretical pulsational instability and behaviour of chemically peculiar stars.

The aim of the present paper is to analyse the pulsational characteristics of the group of $\lambda$ Bootis stars and to test for the presence of a possible Period-Luminosity-Colour-Metallicity relation. The latter is especially interesting in the light of the models by Turcotte et al. (2000). The pulsational characteristics of the $\lambda$ Bootis group (e.g. ratio of variable to non-variable objects and distribution of pulsational constants) may help to put tighter constraints on these models.

\section{Program stars, observations and reductions}

Since our previous works (Paunzen et al. 1997, 1998) several then-selected group members were investigated with classification resolution spectroscopy and found to be misclassified. These are: HD 66920, HD 79025, HD 82573, HD 141851, HD 143148, HD 145782, HD 149303, HD 179791, HD 188164 and HD 192424 (Paunzen 2001). In total, 65 members were selected from the lists of Gray \& Corbally (1993) and Paunzen (2001) which contain well established as well as good candidate $\lambda$ Bootis type objects. Together with the two newly discovered objects (HD 42503 and HD 213669; Sect. 2.3.1), we have a sample of $67 \lambda$ Bootis type stars.

The photometric observations were performed as described by Paunzen et al. (1998) using photoelectric detectors (except for Ref. "4", Table 1, for which a CCD was used) and (if possible) two comparison stars. A standard reduction method for dealing with dead-time, dark counts and tube drifts was applied. The sky measurements (typically one per half hour) were subtracted and differential light curves were generated. For the reduction of the CCD frames for HD 290492 the standard SAAO reduction package as well as the program MOMF (Kjeldsen \& Frandsen 1992) were used. Figure 1 shows light curves of some of our variable program stars.

Frequencies and amplitudes for the variable program stars (listed in Table 2) were derived using a standard Fourier
Table 1. Sites, dates and telescopes used for our survey.

\begin{tabular}{lcccc}
\hline \hline Site & Date & Telescope & Stars & Ref. \\
\hline APT (Fairborn) & 05.2001 & 0.75 & 3 & 1 \\
SAAO & 04.2001 & 0.50 & 8 & 2 \\
& 07.2001 & & & \\
& 08.2001 & & & \\
& 09.2001 & & & \\
& 10.2001 & & & \\
SAAO & 12.2000 & 0.75 & 9 & 3 \\
& 01.2001 & & & \\
SAAO & 08.2001 & 1.00 & 1 & 4 \\
Siding Spring & 01.2002 & 0.60 & 1 & 5 \\
\hline
\end{tabular}

algorithm (Deeming 1975). An analysis with the PhaseDispersion-Minimization (Stellingwerf 1978) gave essentially the same results. A star is considered to be constant, if the Fourier spectrum of the differential light curve does not contain a statistically significant peak (Paunzen et al. 1997). These objects are listed in Table 3.

\subsection{Previously known pulsating $\lambda$ Bootis stars}

The following nine stars were already known as variable. With the only exception of HD 75654 (see below), they have not been re-observed by us:

- HD 6870: Breger (1979) lists a period of 94 min and an amplitude of 15 mmag for Johnson [ $V]$.

- HD 11413: This star is multiperiodic (Waelkens \& Rufener 1983) with a dominant frequency of $54 \mathrm{~min}$ and an amplitude of $18 \mathrm{mmag}$. New measurements (Koen, private communication) confirm the multiperiodic pulsations of this object.

- HD 15165: The multiperiodicity of VW Arietis motivated the fifth STEPHI campaign (Liu et al. 1996). Seven significant periods between 1.8 and $3.9 \mathrm{hr}$ were found in a data set of about $150 \mathrm{hr}$.

- HD 75654: This object was discovered as a $\delta$ Scuti type pulsator by Balona (1977), who reported a period of $0.087 \mathrm{~d}$ $\left(11.49 \mathrm{~d}^{-1}\right)$. Since no other photometric measurements were published, we decided to re-observe this object. We find two frequencies $\left(14.80\right.$ and $\left.15.99 \mathrm{~d}^{-1}\right)$ based on observations during six nights.

- HD 87271: The suspected variability of this star (Handler 2002) was confirmed by Handler et al. (2000). Although the measured light curve spans only several hours, multiperiodicity with a time scale of about $80 \mathrm{~min}$ is evident.

- HD 105759: Martinez et al. (1998) performed a multisite campaign, detecting five pulsation periods between 1.0 and $2.8 \mathrm{hr}$, as well as a detailed abundance analysis for this star.

- HD 110377: Radial velocity variations as well as multiperiodicity with periods between 0.5 and $2.0 \mathrm{hr}$ were reported by Bartolini et al. (1980b). Evidence for amplitude and frequency variations makes this object very interesting for detailed follow-up investigations. Such observations are however beyond the scope of this paper. 


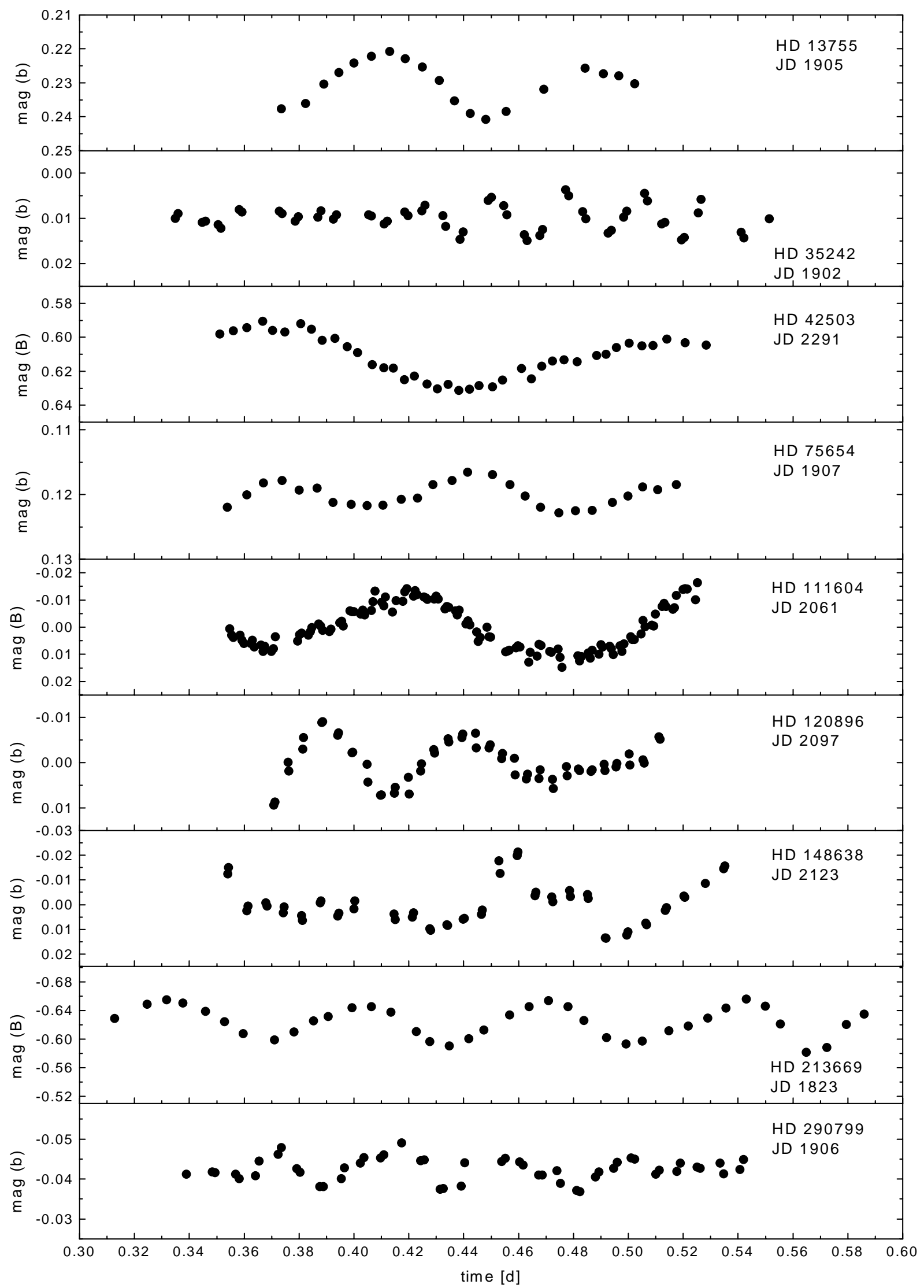

Fig. 1. Differential light curves of eight newly discovered and one confirmed (HD 75654) pulsating $\lambda$ Bootis stars in Strömgren $b$ and Johnson $B$; the dates of the corresponding nights are given as JD $2450000+$ (Table 2). 
Table 2. Observing log of eight newly discovered and one confirmed (HD 75654) pulsating $\lambda$ Bootis stars. Some information on the comparison stars is also given. The differential light curves are shown in Fig. 1.

\begin{tabular}{|c|c|c|c|c|c|c|c|}
\hline HD & JD & hrs & $\begin{array}{c}m_{V} \\
{[\mathrm{mag}]}\end{array}$ & Spec. & $\begin{array}{l}\text { Freq. } \\
{\left[\mathrm{d}^{-1}\right]}\end{array}$ & $\begin{array}{l}\text { Amp. } \\
\text { [mag] }\end{array}$ & Ref. \\
\hline \multirow[t]{4}{*}{13755} & 2451899 & 3.2 & 7.84 & $\lambda \mathrm{Boo}$ & 12.50 & 0.015 & 3 \\
\hline & 2451903 & 2.5 & & & 16.85 & 0.007 & \\
\hline & 2451905 & 3.1 & & & & & \\
\hline & 2451909 & 3.1 & & & & & \\
\hline 13602 & & & 8.52 & F6 & & & \\
\hline 13710 & & & 8.32 & K5 & & & \\
\hline \multirow[t]{3}{*}{35242} & 2451900 & 2.1 & 6.35 & $\lambda$ Boo & 38.61 & 0.005 & 3 \\
\hline & 2451902 & 5.4 & & & 34.16 & 0.003 & \\
\hline & 2451908 & 3.3 & & & 41.33 & 0.003 & \\
\hline 35134 & & & 6.74 & A0 & & & \\
\hline 34888 & & & 6.78 & A5 & & & \\
\hline \multirow[t]{2}{*}{42503} & 2452291 & 4.2 & 7.45 & $\lambda$ Boo & 7.00 & 0.015 & 5 \\
\hline & 2452292 & 1.9 & & & & & \\
\hline 42058 & & & 6.99 & A0 & & & \\
\hline 43452 & & & 7.71 & F5 & & & \\
\hline \multirow[t]{6}{*}{75654} & 2451898 & 3.0 & 6.38 & $\lambda$ Boo & 14.80 & 0.005 & 3 \\
\hline & 2451902 & 1.8 & & & 15.99 & 0.002 & \\
\hline & 2451905 & 3.1 & & & & & \\
\hline & 2451906 & 1.2 & & & & & \\
\hline & 2451907 & 3.8 & & & & & \\
\hline & 2451909 & 3.6 & & & & & \\
\hline 74978 & & & 6.87 & A1 & & & \\
\hline 75272 & & & 6.98 & B9.5 & & & \\
\hline 111604 & 2452061 & 4.1 & 5.89 & $\lambda$ Boo & 8.77 & 0.020 & 1 \\
\hline 112412 & & & 5.61 & F1 & & & \\
\hline 110375 & & & 8.33 & F5 & & & \\
\hline 120896 & 2452097 & 3.9 & 8.50 & $\lambda$ Boo & 17.79 & 0.010 & 2 \\
\hline 121372 & & & 8.67 & G5 & & & \\
\hline \multirow[t]{2}{*}{148638} & 2452097 & 4.6 & 7.90 & $\lambda$ Boo & 16.32 & 0.016 & 2 \\
\hline & 2452123 & 5.0 & & & & & \\
\hline 148596 & & & 8.60 & $\mathrm{~F} 2$ & & & \\
\hline 148573 & & & 8.63 & B9 & & & \\
\hline \multirow[t]{3}{*}{213669} & 2451823 & 6.5 & 7.42 & $\lambda$ Boo & 15.01 & 0.023 & 2 \\
\hline & 2451826 & 1.6 & & & & & \\
\hline & 2451827 & 1.1 & & & & & \\
\hline 211878 & & & 7.70 & F5 & & & \\
\hline 214390 & & & 7.90 & F3 & & & \\
\hline \multirow[t]{2}{*}{290799} & 2451904 & 3.0 & 10.63 & $\lambda$ Boo & 23.53 & 0.006 & 3 \\
\hline & 2451906 & 4.8 & & & & & \\
\hline 37652 & & & 7.35 & F5 & & & \\
\hline 290798 & & & 10.40 & $\mathrm{~A} 2$ & & & \\
\hline
\end{tabular}

- HD 153747: Desikachary \& McInally (1979) reported multiperiodicity (periods between 0.96 and $1.2 \mathrm{hr}$ ) as well as a variable frequency spectrum of this object. Unfortunately no further references on the pulsational behaviour of this star were found.

- HD 192640: This star's variability was discovered by Gies \& Percy (1977). Since 1995 permanent multisite observations have been performed. No detailed overall analysis has been published to date. Data subsets (Kusakin \& Mkrtichian 1996; Paunzen \& Handler 1996; Mkrtichian et al. 2000) suggest multiperiodicity with a main period of $38 \mathrm{~min}$ and an amplitude of 20 mmag in Johnson $V$.
We have used the published frequencies and amplitudes of these objects from the above-mentioned references in our analysis. If more than one frequency has been published, we have weighted the individual periods with the squared amplitude to obtain a mean period.

\subsection{Group members not observed by us}

Five well established members of the $\lambda$ Bootis group were not photometrically investigated: HD 110411, HD 125889 , HD 170680, HD 184779 and HD 198160. For three of them 
Table 3. Observing log of thirteen $\lambda$ Bootis stars not found to pulsate as well as some comparison star information.

\begin{tabular}{|c|c|c|c|c|c|c|}
\hline $\mathrm{HD} / \mathrm{BD}$ & JD & hrs & $\begin{array}{c}m_{V} \\
{[\mathrm{mag}]}\end{array}$ & Spec. & $\begin{array}{c}\text { Limit } \\
{[\mathrm{mmag}]}\end{array}$ & Ref \\
\hline \multirow[t]{2}{*}{7908} & 2451898 & 2.0 & 7.29 & $\lambda \mathrm{Boo}$ & 0.3 & 3 \\
\hline & 2451907 & 2.3 & & & & \\
\hline 7629 & & & 7.13 & A9 & & \\
\hline 7896 & & & 7.95 & G6 & & \\
\hline 24472 & 2451900 & 2.9 & 7.09 & $\lambda \mathrm{Boo}$ & 0.8 & 3 \\
\hline 24616 & & & 6.70 & G8 & & \\
\hline 25385 & & & 7.40 & F0 & & \\
\hline 54272 & 2451908 & 2.6 & 8.80 & $\lambda$ Boo & 1.4 & 3 \\
\hline 54692 & & & 8.51 & A0 & & \\
\hline+19622 & & & 8.90 & A2 & & \\
\hline 74873 & 2451904 & 3.5 & 5.89 & $\lambda \mathrm{Boo}$ & 1.6 & 3 \\
\hline 74228 & & & 5.65 & A3 & & \\
\hline 75108 & & & 8.38 & G5 & & \\
\hline 83277 & 2451901 & 3.5 & 8.30 & $\lambda$ Boo & 1.4 & 3 \\
\hline 83547 & & & 8.62 & A0 & & \\
\hline 82709 & & & 8.04 & A9 & & \\
\hline 90821 & 2452039 & 2.0 & 9.47 & $\lambda$ Boo & 2.2 & 1 \\
\hline 90878 & & & 7.82 & F8 & & \\
\hline 90748 & & & 8.67 & F8 & & \\
\hline 107223 & 2452003 & 5.2 & 7.35 & $\lambda$ Boo & 1.9 & 2 \\
\hline 107143 & & & 7.87 & A1 & & \\
\hline 107265 & & & 8.76 & A0 & & \\
\hline 111005 & 2452004 & 1.9 & 7.96 & $\lambda$ Boo & 2.1 & 2 \\
\hline 110705 & & & 8.36 & F0 & & \\
\hline 110989 & & & 8.41 & F8 & & \\
\hline 130767 & 2452039 & 5.5 & 6.91 & $\lambda$ Boo & 1.2 & 1 \\
\hline 130556 & & & 7.84 & $\mathrm{~F} 1$ & & \\
\hline 130396 & & & 7.41 & F8 & & \\
\hline 149130 & 2452127 & 5.6 & 8.50 & $\lambda$ Boo & 2.4 & 2 \\
\hline 148597 & & & 8.25 & B9 & & \\
\hline 149471 & & & 8.94 & F6 & & \\
\hline \multirow[t]{2}{*}{216847} & 2452190 & 2.9 & 7.06 & $\lambda$ Boo & 1.7 & 2 \\
\hline & 2452191 & 3.2 & & & & \\
\hline 216349 & & & 7.84 & K1 & & \\
\hline 217686 & & & 7.56 & F7 & & \\
\hline \multirow[t]{3}{*}{290492} & 2451901 & 3.0 & 9.27 & $\lambda$ Boo & 1.8 & 2 \\
\hline & 2452190 & 2.2 & & & & \\
\hline & 2452192 & 4.0 & & & & \\
\hline 290575 & & & 9.85 & F5 & & \\
\hline-00984 & & & 8.37 & $\mathrm{HgMn}$ & & \\
\hline \multirow[t]{2}{*}{261904} & 2452190 & 2.1 & 10.20 & $\lambda$ Boo & 3.5 & 4 \\
\hline & 2452191 & 2.2 & & & & \\
\hline 261941 & & & 10.94 & $\mathrm{~A} 2$ & & \\
\hline
\end{tabular}

(HD 110411, HD 170680 and HD 198160) photometric measurements in the HIPPARCOS and TYCHO catalogues (ESA 1997) were found. Since these observations are not optimal to find $\delta$ Scuti type pulsation, only a rough estimate for variability can be made. We find a level of non-variability based on the HIPPARCOS photometry of 3 mmag for HD 110411 and HD 170680, and 4 mmag for HD 198160. In fact, HD 110411 was suspected as variable by Bartolini et al. (1980a), but Antonello \& Mantegazza (1982) concluded that there is no evidence for periodic terms in the light curve: different oscillation modes may be excited occasionally and then be damped again. We therefore treat this star as being constant within a limit of 3 mmag. Consequently, the other two objects (HD 125889 and HD 184779) were not considered in the following analysis.

\subsection{Notes on individual stars}

In the following sections we describe special properties of some individual stars in more detail.

\subsubsection{HD 42503 and HD 213669}

These two objects were suspected $\delta$ Scuti type pulsators based on HIPPARCOS photometry (Handler 2002). Handler (1999) presented Strömgren $u v b y \beta$ photometry which puts these stars well within the typical area of the $\lambda$ Bootis objects in a $m_{1}$ versus $(b-y)$ diagram (Paunzen et al. 1998). Our photometric measurements confirmed the pulsation.

We have performed additional spectroscopic observations to establish the nature of these stars. These observations were done on the $1.9 \mathrm{~m}$ telescope at SAAO in the night of 03./04.10.2000. The Grating Spectrograph with the SITe CCD together with the 600 lines $\mathrm{mm}^{-1}$ grating resulted in a useful wavelength range of $1600 \AA$, a resolution of $2 \AA$ and a signal-to-noise ratio of about 200. The wavelength calibration was done with the help of a CuAr lamp within standard IRAF routines. The classification was done within the system described by Paunzen (2001). Both objects are very good $\lambda$ Bootis candidates, with derived spectral types of A2 V ( $\lambda$ Boo) and kA1hF0mA1 V $\lambda$ Boo for HD 42503 and HD 213669, respectively. The notation of the spectral classification is according to Gray (1988) where $k$ stands for the classification of the Ca II K line, $h$ for the hydrogen lines and $m$ for the appearance of the metallic-line spectrum compared to MK standards. We therefore included them in our sample. Figure 2 shows their spectra together with those of two well established $\lambda$ Bootis stars (HD 107233 and HD 198160; taken from Paunzen 2001) which exhibit similar spectral characteristics. However, we note that a final decision on their group membership has to be made after a detailed determination of their chemical abundances (especially of C, N, O and S) which was not done so far.

\subsubsection{HD 64491 and HD 111786}

Both stars are $\delta$ Scuti type pulsators and were reported as well established members of the group by Gray (1988) and Paunzen \& Gray (1997). However, both objects are spectroscopic binary systems (Faraggiana \& Bonifacio 1999; Iliev et al. 2001). The published information on these stars does not allow us to decide whether they are true $\lambda$ Bootis type objects, thus we have not included either of them in our sample. 


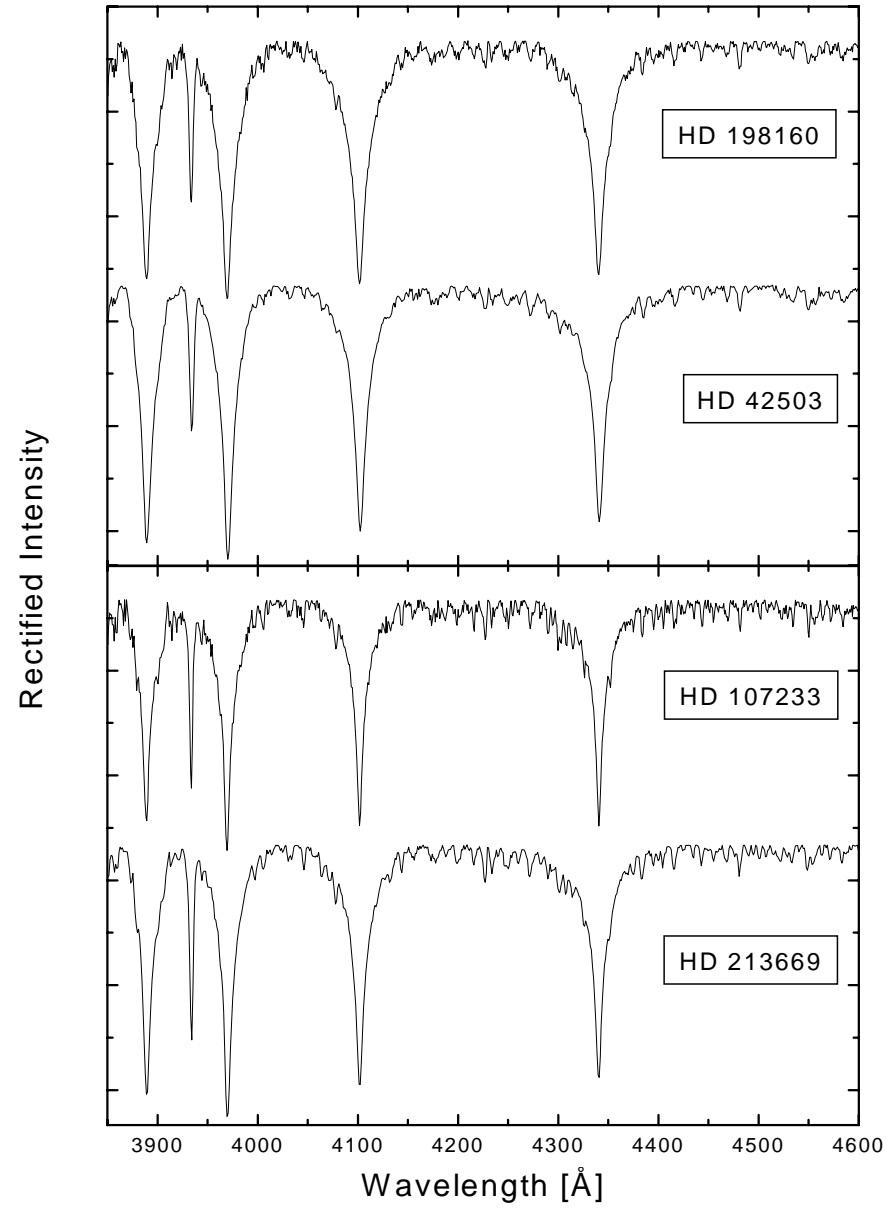

Fig. 2. Classification resolution spectra of the two newly discovered $\lambda$ Bootis candidates HD 42503 (A2 V $\lambda$ Boo; upper panel) and HD 213669 (kA1hF0mA1 V $\lambda$ Boo; lower panel) together with the two well established objects HD 107233 (kA1hF0mA1 V $\lambda$ Boo) and HD 198160 (A2 Vann $\lambda$ Boo) for comparison.

\subsubsection{HD 74873}

We have re-observed this object because the upper limit for non-variability given by Paunzen et al. (1997) was very high (9.4 mmag). Our new observations showed no variability within a limit of $1.6 \mathrm{mmag}$.

\subsubsection{HD 175445}

This object exhibits a peculiar behaviour which we have, so far, been unable to understand. During our first short observing run (on 06/07 July 2001), its magnitude and colour were consistent with their standard values from the literature $(b=7.85$, $v-b=0.222$ ), and marginal evidence for pulsational variability was found. However, when we attempted to re-observe the star on 05/06 August 2001, it appeared much fainter and redder than in the previous month.

Consequently, we double-checked its correct identification and re-examined the literature. We found no evidence for a misidentification or previous peculiar behaviour. We obtained a $3 \mathrm{hr}$ light curve on the night of 06/07 August 2001, where the object appeared as a star of $b=10.67$ and $v-b=1.17$. These observations are consistent with an eclipse by a late K subgiant companion. However, the evolutionary history of such a system would require the binary to be close, and no other possible eclipses have yet been reported.

\subsubsection{HD 290799}

Paunzen et al. (1998) reported this star as constant with an upper limit of 10 mmag in Geneva $V_{1}$. Our new observations show the star to be pulsating with a frequency of $23.53 \mathrm{~d}^{-1}$ and an amplitude of 6 mmag in Strömgren $b$.

\section{Basic stellar parameters}

In this section we describe the calibration procedures within various photometric systems and derivation of the basic stellar parameters required to analyse the pulsational characteristics of these stars, such as the effective temperature, surface gravity and the luminosity.

The required standard photometric colours were taken from the General Catalogue of Photometric Data (GCPD; http://obswww. unige.ch/gcpd/) as well as the HIPPARCOS and TYCHO databases (ESA 1997). If available, averaged and weighted mean values were used.

The following calibrations for the individual photometric systems were used to derive effective temperatures and surface gravities:

- Johnson $U B V$ : Napiwotzki et al. (1993);

- Strömgren uvbyß: Moon \& Dworetsky (1985) and Napiwotzki et al. (1993);

- Geneva 7-colour: Kobi \& North (1990) and Künzli et al. (1997).

The calibrations for the Johnson $U B V$ and Geneva 7-colour system need an a-priori knowledge of the reddening, which is, in general, not easy to estimate.

Normally, the reddening for objects within the solar neighborhood is estimated using photometric calibrations in the Strömgren $u v b y \beta$ system (Strömgren 1966; Crawford 1979; Hilditch et al. 1983). These calibrations are not very reliable for stars with spectral types from A0 to A3 (Gerbaldi et al. 1999), mainly because for these stars, the reddening free parameter $\beta$ is no longer a temperature indicator alone but is also sensitive to the luminosity. From the photometry we find that two of our pulsating (HD 125162 and HD 183324) and nine constant (HD 23392, HD 31295, HD 36726, HD 74873, HD 110411, HD 130767, HD 170680, HD 261904 and HD 294253) program stars fall into A0 to A3 spectral region.

An independent way to derive the interstellar reddening is to use galactic reddening maps, which are derived from open clusters as well as from galactic field stars. Several different models have been published in the literature (Arenou et al. 1992; Hakkila et al. 1997). Chen et al. (1998) compared the results from Arenou et al. (1992) and those derived from the HIPPARCOS measurements and found an overestimation of previously published results from Arenou et al. (1992) for distances less than $500 \mathrm{pc}$. They consequently proposed a new 
Table 4. Pulsating $\lambda$ Bootis stars; an asterisk denotes stars without an accurate HIPPARCOS parallax; $\sigma(b-y)= \pm 0.005$ mag. In parenthesis are the errors in the final digits of the corresponding quantity.

\begin{tabular}{|c|c|c|c|c|c|c|c|c|c|c|}
\hline HD & $\begin{array}{c}(b-y)_{0} \\
{[\mathrm{mag}]}\end{array}$ & $\begin{array}{c}\log T_{\text {eff }} \\
\text { [dex] }\end{array}$ & $\begin{array}{l}\log g \\
{[\operatorname{dex}]}\end{array}$ & $\begin{array}{c}v \sin i \\
{\left[\mathrm{~km} \mathrm{~s}^{-1}\right]}\end{array}$ & $\begin{array}{c}{[Z]} \\
{[\operatorname{dex}]}\end{array}$ & $\begin{array}{c}M_{V} \\
{[\mathrm{mag}]}\end{array}$ & $\begin{array}{c}M_{B} \\
{[\mathrm{mag}]}\end{array}$ & $\begin{array}{c}\log L_{*} / L_{\odot} \\
{[\operatorname{dex}]}\end{array}$ & $\log P$ & $\begin{array}{c}Q \\
{[\mathrm{~d}]}\end{array}$ \\
\hline 6870 & 0.164 & $3.865(6)$ & $3.84(11)$ & 165 & $-1.03(20)$ & $+2.29(42)$ & +2.20 & $1.02(17)$ & -1.19 & 0.023 \\
\hline 11413 & 0.104 & $3.899(7)$ & $3.91(21)$ & 125 & $-1.17(10)$ & $+1.49(10)$ & +1.36 & $1.35(4)$ & -1.38 & 0.014 \\
\hline 13755 & 0.181 & $3.850(10)$ & $3.26(10)$ & - & $-0.75(30)$ & $+0.93(10)$ & +0.83 & $1.57(4)$ & -1.12 & 0.010 \\
\hline 15165 & 0.189 & $3.846(12)$ & $3.23(10)$ & 90 & $-1.15(17)$ & $+1.12(16)$ & +1.01 & $1.50(6)$ & -0.87 & 0.017 \\
\hline 30422 & 0.098 & $3.896(6)$ & $4.00(20)$ & 135 & $-1.50(20)$ & $+2.35(2)$ & +2.23 & $1.01(1)$ & -1.68 & 0.010 \\
\hline 35242 & 0.058 & $3.916(5)$ & $3.90(14)$ & 90 & $-1.40(20)$ & $+1.75(22)$ & +1.60 & $1.26(9)$ & -1.58 & 0.010 \\
\hline 42503 & 0.110 & $3.885(16)$ & $3.10(10)$ & - & $-0.83(20)$ & $-0.03(4)$ & -0.14 & $1.96(2)$ & -0.85 & 0.013 \\
\hline 75654 & 0.158 & $3.866(6)$ & $3.77(11)$ & 45 & $-0.91(11)$ & $+1.83(12)$ & +1.74 & $1.20(5)$ & -1.18 & 0.019 \\
\hline 83041 & 0.185 & $3.852(13)$ & $3.76(20)$ & 95 & $-1.03(8)$ & $+1.70(30)$ & +1.60 & $1.26(12)$ & -1.18 & 0.018 \\
\hline $84948 B^{*}$ & 0.196 & $3.833(13)$ & $3.70(15)$ & 55 & $-0.82(19)$ & $+1.63(30)$ & +1.75 & $1.20(12)$ & -1.11 & 0.020 \\
\hline 87271 & 0.149 & $3.876(13)$ & $3.43(10)$ & - & $-1.11(30)$ & $+1.02(8)$ & +0.92 & $1.53(3)$ & -1.27 & 0.009 \\
\hline 102541 & 0.141 & $3.885(10)$ & $4.22(16)$ & - & $-0.95(20)$ & $+2.34(21)$ & +2.23 & 1.01(9) & -1.30 & 0.029 \\
\hline 105058 & 0.127 & $3.889(10)$ & $3.77(30)$ & 140 & $-0.82(7)$ & $+0.86(30)$ & +0.75 & $1.60(12)$ & -1.40 & 0.010 \\
\hline 105759 & 0.142 & $3.874(6)$ & $3.65(10)$ & 120 & $-0.92(30)$ & $+1.35(21)$ & +1.25 & $1.40(8)$ & -1.20 & 0.015 \\
\hline $109738^{*}$ & 0.144 & $3.881(8)$ & $3.90(13)$ & - & $-1.02(20)$ & $+1.85(30)$ & +1.75 & $1.20(12)$ & -1.49 & 0.012 \\
\hline 110377 & 0.120 & $3.888(5)$ & $3.97(14)$ & 170 & $-0.83(20)$ & $+1.96(11)$ & +1.85 & $1.16(5)$ & -1.45 & 0.014 \\
\hline 111604 & 0.112 & $3.890(8)$ & $3.61(25)$ & 180 & $-1.04(3)$ & $+0.48(7)$ & +0.37 & $1.75(3)$ & -0.94 & 0.022 \\
\hline 120500 & 0.064 & $3.915(4)$ & $3.86(10)$ & 125 & $-0.73(14)$ & $+0.85(34)$ & +0.70 & $1.62(13)$ & -1.32 & 0.014 \\
\hline $120896^{*}$ & 0.166 & $3.861(5)$ & $3.76(10)$ & - & $-0.82(30)$ & $+1.90(30)$ & +1.81 & $1.18(12)$ & -1.25 & 0.016 \\
\hline 125162 & 0.042 & $3.941(8)$ & $4.07(9)$ & 115 & $-1.61(24)$ & $+1.71(23)$ & +1.54 & $1.28(9)$ & -1.64 & 0.011 \\
\hline 142703 & 0.177 & $3.861(9)$ & $3.93(12)$ & 100 & $-1.32(5)$ & $+2.41(12)$ & +2.32 & $0.97(5)$ & -1.43 & 0.015 \\
\hline $142944^{*}$ & 0.198 & $3.845(8)$ & $3.19(4)$ & 180 & $-0.91(38)$ & $+0.80(30)$ & +0.69 & $1.62(12)$ & -0.85 & 0.016 \\
\hline $148638^{*}$ & 0.106 & $3.882(13)$ & $3.39(10)$ & - & $-0.80(30)$ & $+0.33(30)$ & +0.23 & $1.81(12)$ & -1.21 & 0.009 \\
\hline 153747 & 0.068 & $3.914(5)$ & $3.70(24)$ & - & $-0.86(20)$ & $+1.24(30)$ & +1.09 & $1.46(12)$ & -1.31 & 0.013 \\
\hline 168740 & 0.128 & $3.883(5)$ & $3.88(14)$ & 145 & $-0.91(8)$ & $+1.82(2)$ & +1.72 & $1.21(1)$ & -1.44 & 0.013 \\
\hline $168947^{*}$ & 0.145 & $3.878(11)$ & $3.67(10)$ & - & $-0.74(20)$ & $+1.28(30)$ & +1.18 & $1.43(12)$ & -1.23 & 0.014 \\
\hline 183324 & 0.032 & $3.952(10)$ & $4.13(4)$ & 90 & $-1.47(6)$ & $+1.64(42)$ & +1.44 & $1.32(17)$ & -1.68 & 0.011 \\
\hline $191850^{*}$ & 0.163 & $3.869(9)$ & $3.61(10)$ & - & $-0.96(30)$ & $+1.50(30)$ & +1.41 & $1.34(12)$ & -1.13 & 0.017 \\
\hline 192640 & 0.095 & $3.900(5)$ & $3.95(18)$ & 80 & $-1.46(8)$ & $+1.84(2)$ & +1.71 & $1.22(1)$ & -1.55 & 0.011 \\
\hline 210111 & 0.136 & $3.878(7)$ & $3.84(15)$ & 55 & $-1.04(20)$ & $+1.76(15)$ & +1.66 & $1.23(6)$ & -1.36 & 0.014 \\
\hline 213669 & 0.155 & $3.872(8)$ & $3.82(17)$ & - & $-0.93(20)$ & $+1.79(21)$ & +1.69 & $1.22(8)$ & -1.18 & 0.021 \\
\hline 221756 & 0.046 & $3.930(10)$ & $3.90(3)$ & 105 & $-0.71(3)$ & $+1.16(16)$ & +1.00 & $1.50(6)$ & -1.36 & 0.015 \\
\hline $290799^{*}$ & 0.114 & $3.889(5)$ & $4.18(10)$ & 70 & $-0.82(26)$ & $+2.62(30)$ & +2.51 & $0.90(12)$ & -1.37 & 0.025 \\
\hline
\end{tabular}

model for galactic latitudes of $\pm 10^{\circ}$, but otherwise find excellent agreement with the model by Sandage (1972). We have used the proposed model by Chen et al. (1998) to derive the interstellar reddening for all program stars. The values from the calibration of the Strömgren $u v b y \beta$ and the model by Chen et al. (1998) are in very good agreement. To minimize possible inconsistencies we have averaged the values from both approaches.

In Table 7, we compare our photometrically derived effective temperatures and surface gravities of 29 program stars from this work (TW) with those of Table 1 from Heiter et al. (2002), which contains averaged values from the literature based on spectroscopic analyses. The average difference for the effective temperature is $\Delta T_{\text {eff }}=T_{\text {eff }}($ Lit. $)-T_{\text {eff }}(\mathrm{TW})=$ $+72(210) \mathrm{K}$, and for the average surface gravity $\Delta \log g=$ $\log g$ (Lit. $)-\log g(\mathrm{TW})=+0.07(24)$ dex. We note that there are some stars for which the spectroscopically derived values are significantly different from the photometrically derived ones (e.g. HD 106223 and HD 107233). These cases were already extensively discussed by Heiter et al. (2002). Although such deviating cases obviously exist, we believe that our calibration method is consistent and therefore suitable for a statistical analysis.

For all program stars photometrically calibrated absolute magnitudes (assuming that all objects are single) were estimated with an error of $\pm 0.3 \mathrm{mag}$. As an independent source we have taken the HIPPARCOS parallaxes (if available) to derive absolute magnitudes using the visual magnitude and reddening. Since we also corrected for the Lutz-Kelker effect (Koen 1992) which is only possible for parallax measurements with an absolute error of $[\sigma(\pi) / \pi]<0.175$ it seriously limits the useful data. Oudmaijer et al. (1998) showed that this effect has to be taken into account if individual absolute magnitudes are calculated using HIPPARCOS parallaxes. Stars without measurements satisfying $[\sigma(\pi) / \pi]<0.175$ are marked with an asterisk in Tables 4 and 6 (20 stars in total). For the other 45 objects we are able to derive weighted means (taking the errors as weights, i.e. a larger error is a lower weight) for the absolute magnitude using the values from the photometric calibration procedure and the conversion of the HIPPARCOS parallax measurements. 
Table 5. $\delta$ Scuti stars selected from the list by Rodriguez et al. (2000); $\sigma(b-y)= \pm 0.005$ mag; $\sigma[Z]= \pm 0.15$ dex. In parenthesis are the errors in the final digits of the corresponding quantity.

\begin{tabular}{|c|c|c|c|c|c|c|c|c|c|c|}
\hline HD & $\begin{array}{c}(b-y)_{0} \\
{[\mathrm{mag}]}\end{array}$ & $\begin{array}{c}\log T_{\text {eff }} \\
\text { [dex] }\end{array}$ & $\begin{array}{l}\log g \\
{[\operatorname{dex}]}\end{array}$ & $\begin{array}{c}v \sin i \\
{\left[\mathrm{~km} \mathrm{~s}^{-1}\right]}\end{array}$ & $\begin{array}{c}{[Z]} \\
{[\mathrm{dex}]}\end{array}$ & $\begin{array}{c}M_{V} \\
{[\mathrm{mag}]}\end{array}$ & $\begin{array}{c}M_{B} \\
{[\mathrm{mag}]}\end{array}$ & $\begin{array}{c}\log L_{*} / L_{\odot} \\
{[\mathrm{dex}]}\end{array}$ & $\log P$ & $\begin{array}{c}Q Q \\
{[\mathrm{~d}]}\end{array}$ \\
\hline 432 & 0.211 & $3.841(4)$ & $3.44(7)$ & 70 & +0.45 & $1.19(29)$ & 1.08 & $1.47(11)$ & -1.00 & 0.017 \\
\hline 3112 & 0.127 & $3.883(5)$ & $3.59(9)$ & 80 & +0.28 & $0.54(84)$ & 0.44 & $1.73(34)$ & -1.31 & 0.009 \\
\hline 4490 & 0.156 & $3.867(3)$ & $3.55(9)$ & 180 & +0.22 & $0.92(15)$ & 0.83 & $1.57(6)$ & -0.98 & 0.019 \\
\hline 4849 & 0.168 & $3.862(2)$ & $3.78(8)$ & - & +0.52 & $1.65(30)$ & 1.56 & $1.27(12)$ & -1.26 & 0.016 \\
\hline 7312 & 0.169 & $3.861(3)$ & $3.79(6)$ & - & +0.24 & $1.71(28)$ & 1.62 & $1.25(11)$ & -1.38 & 0.012 \\
\hline 8511 & 0.134 & $3.880(4)$ & $3.96(6)$ & 190 & -0.06 & $2.04(1)$ & 1.94 & $1.12(1)$ & -1.16 & 0.027 \\
\hline 8781 & 0.213 & $3.838(6)$ & $3.46(6)$ & - & -0.03 & $1.57(18)$ & 1.46 & $1.32(7)$ & -0.95 & 0.020 \\
\hline 9065 & 0.200 & $3.844(6)$ & $3.46(6)$ & - & -0.14 & $1.72(21)$ & 1.61 & $1.26(8)$ & -1.02 & 0.018 \\
\hline 9100 & 0.087 & $3.899(8)$ & $3.53(22)$ & 120 & -0.34 & $0.43(28)$ & 0.30 & $1.78(11)$ & -0.87 & 0.024 \\
\hline 11522 & 0.162 & $3.861(5)$ & $3.41(6)$ & 120 & -0.04 & $0.76(13)$ & 0.67 & $1.63(5)$ & -1.04 & 0.014 \\
\hline 15550 & 0.152 & $3.871(3)$ & $3.84(6)$ & 170 & +0.13 & $1.89(1)$ & 1.80 & $1.18(1)$ & -1.17 & 0.022 \\
\hline 15634 & 0.179 & $3.858(2)$ & $3.81(7)$ & 140 & +0.28 & $1.57(50)$ & 1.48 & $1.31(20)$ & -1.01 & 0.028 \\
\hline 17093 & 0.133 & $3.883(8)$ & $4.04(11)$ & 75 & -0.10 & $2.22(4)$ & 2.12 & $1.05(2)$ & -1.45 & 0.016 \\
\hline 19279 & 0.063 & $3.913(8)$ & $3.76(17)$ & 285 & -0.16 & $1.69(61)$ & 1.54 & $1.28(25)$ & -1.16 & 0.022 \\
\hline 23728 & 0.178 & $3.859(5)$ & $3.70(16)$ & 105 & -0.21 & $1.62(15)$ & 1.53 & $1.29(6)$ & -1.00 & 0.025 \\
\hline 24809 & 0.119 & $3.890(10)$ & $4.26(20)$ & 130 & -0.36 & $2.51(7)$ & 2.40 & $0.94(3)$ & -1.26 & 0.035 \\
\hline 24832 & 0.158 & $3.865(4)$ & $3.69(13)$ & 140 & +0.12 & $1.12(30)$ & 1.03 & $1.49(12)$ & -0.81 & 0.035 \\
\hline 26574 & 0.196 & $3.847(4)$ & $3.49(12)$ & 100 & +0.62 & $1.22(38)$ & 1.11 & $1.46(15)$ & -1.13 & 0.013 \\
\hline 27397 & 0.166 & $3.864(3)$ & $3.96(5)$ & 100 & +0.22 & $2.30(5)$ & 2.21 & $1.02(2)$ & -1.26 & 0.022 \\
\hline 27459 & 0.123 & $3.884(5)$ & $3.96(13)$ & 75 & +0.25 & $1.92(21)$ & 1.82 & $1.17(9)$ & -1.44 & 0.014 \\
\hline 28024 & 0.159 & $3.865(7)$ & $3.40(18)$ & 210 & +0.22 & $0.77(24)$ & 0.68 & $1.63(9)$ & -0.83 & 0.022 \\
\hline 28319 & 0.093 & $3.901(9)$ & $3.70(12)$ & 80 & +0.16 & $0.32(78)$ & 0.19 & $1.82(31)$ & -1.12 & 0.016 \\
\hline 28910 & 0.139 & $3.877(4)$ & $3.97(5)$ & 125 & +0.19 & $1.58(75)$ & 1.48 & $1.31(30)$ & -1.17 & 0.024 \\
\hline 30780 & 0.114 & $3.887(3)$ & $3.87(11)$ & 150 & +0.23 & $1.41(50)$ & 1.30 & $1.38(20)$ & -1.38 & 0.013 \\
\hline 32846 & 0.189 & $3.845(9)$ & $3.37(10)$ & - & -0.19 & $1.16(5)$ & 1.05 & $1.48(2)$ & -0.87 & 0.021 \\
\hline 50018 & 0.217 & $3.836(6)$ & $3.35(10)$ & 135 & +0.78 & $0.49(1.03)$ & 0.37 & $1.75(41)$ & -0.81 & 0.019 \\
\hline 57167 & 0.214 & $3.844(2)$ & $3.97(3)$ & 100 & +0.18 & $2.49(15)$ & 2.38 & $0.95(6)$ & -1.33 & 0.019 \\
\hline 71496 & 0.133 & $3.878(4)$ & $3.61(8)$ & 130 & +0.41 & $1.18(10)$ & 1.08 & $1.47(4)$ & -1.02 & 0.021 \\
\hline 71935 & 0.140 & $3.872(4)$ & $3.67(9)$ & 160 & +0.32 & $1.14(20)$ & 1.04 & $1.49(8)$ & -1.15 & 0.016 \\
\hline 73575 & 0.137 & $3.874(5)$ & $3.41(12)$ & 150 & +0.32 & $0.38(21)$ & 0.28 & $1.79(8)$ & -0.99 & 0.015 \\
\hline 74050 & 0.106 & $3.892(7)$ & $3.85(16)$ & 145 & +0.25 & $1.71(44)$ & 1.59 & $1.26(18)$ & -1.24 & 0.019 \\
\hline 84999 & 0.192 & $3.851(4)$ & $3.41(8)$ & 110 & +0.13 & $1.09(27)$ & 0.99 & $1.50(11)$ & -0.88 & 0.021 \\
\hline 88824 & 0.153 & $3.870(2)$ & $3.83(10)$ & 235 & +0.08 & $1.76(2)$ & 1.67 & $1.23(1)$ & -0.90 & 0.039 \\
\hline 94985 & 0.088 & $3.903(9)$ & $3.62(5)$ & - & -0.11 & $0.82(16)$ & 0.69 & $1.62(6)$ & -0.82 & 0.032 \\
\hline 103313 & 0.110 & $3.889(4)$ & $3.67(8)$ & 70 & +0.24 & $0.79(36)$ & 0.68 & $1.63(14)$ & -1.10 & 0.017 \\
\hline 104036 & 0.086 & $3.899(7)$ & $4.09(14)$ & - & +0.01 & $1.67(40)$ & 1.54 & $1.28(16)$ & -1.52 & 0.013 \\
\hline 107131 & 0.097 & $3.897(5)$ & $4.03(20)$ & 185 & -0.08 & $1.91(25)$ & 1.78 & $1.19(10)$ & -1.18 & 0.029 \\
\hline 107904 & 0.224 & $3.837(6)$ & $3.20(12)$ & 115 & +0.68 & $0.83(15)$ & 0.72 & $1.61(6)$ & -0.93 & 0.013 \\
\hline 109585 & 0.208 & $3.841(4)$ & $3.59(10)$ & 80 & +0.16 & $1.80(18)$ & 1.69 & $1.22(7)$ & -1.09 & 0.018 \\
\hline 115308 & 0.199 & $3.846(6)$ & $3.36(8)$ & 75 & +0.06 & $1.16(2)$ & 1.05 & $1.48(1)$ & -0.93 & 0.017 \\
\hline 117661 & 0.095 & $3.900(4)$ & $3.95(11)$ & 55 & +0.14 & $1.70(12)$ & 1.57 & $1.27(5)$ & -1.37 & 0.016 \\
\hline 124675 & 0.111 & $3.884(6)$ & $3.67(17)$ & 120 & -0.11 & $1.02(29)$ & 0.91 & $1.54(12)$ & -1.19 & 0.015 \\
\hline 125161 & 0.128 & $3.885(8)$ & $4.10(19)$ & 135 & +0.08 & $2.40(14)$ & 2.29 & $0.98(5)$ & -1.58 & 0.013 \\
\hline 127762 & 0.112 & $3.890(3)$ & $3.69(12)$ & 130 & +0.02 & $0.94(2)$ & 0.83 & $1.57(1)$ & -1.14 & 0.017 \\
\hline 127929 & 0.143 & $3.876(3)$ & $3.65(11)$ & 70 & +0.00 & $0.80(29)$ & 0.70 & $1.62(12)$ & -1.06 & 0.018 \\
\hline 138918 & 0.146 & $3.870(5)$ & $3.77(23)$ & 85 & +0.16 & $0.19(1.13)$ & 0.10 & $1.86(45)$ & -0.81 & 0.032 \\
\hline 143466 & 0.177 & $3.863(5)$ & $3.92(15)$ & 145 & +0.24 & $2.29(8)$ & 2.20 & $1.02(3)$ & -1.12 & 0.029 \\
\hline 152569 & 0.160 & $3.864(3)$ & $3.81(13)$ & 195 & +0.16 & $1.83(4)$ & 1.74 & $1.21(2)$ & -1.12 & 0.023 \\
\hline 155514 & 0.119 & $3.886(6)$ & $3.90(16)$ & 175 & +0.08 & $1.49(17)$ & 1.38 & $1.35(7)$ & -1.05 & 0.029 \\
\hline 171369 & 0.159 & $3.863(3)$ & $3.79(8)$ & 80 & +0.05 & $1.64(20)$ & 1.55 & $1.28(8)$ & -1.04 & 0.026 \\
\hline 176723 & 0.200 & $3.848(3)$ & $3.62(9)$ & 265 & +0.10 & $1.66(19)$ & 1.56 & $1.27(8)$ & -0.87 & 0.031 \\
\hline 177392 & 0.168 & $3.861(6)$ & $3.54(16)$ & 140 & +0.15 & $0.96(10)$ & 0.87 & $1.55(4)$ & -0.96 & 0.020 \\
\hline 177482 & 0.161 & $3.862(5)$ & $3.45(8)$ & 145 & +0.26 & $0.86(14)$ & 0.77 & $1.59(6)$ & -1.01 & 0.016 \\
\hline 181333 & 0.138 & $3.877(4)$ & $3.53(6)$ & 55 & +0.38 & $0.47(47)$ & 0.37 & $1.75(19)$ & -0.82 & 0.025 \\
\hline 182475 & 0.194 & $3.850(3)$ & $3.63(14)$ & 130 & +0.32 & $1.61(47)$ & 1.51 & $1.30(19)$ & -1.11 & 0.018 \\
\hline
\end{tabular}


Table 5. continued.

\begin{tabular}{ccccccccccc}
\hline \hline HD & $\begin{array}{c}(b-y)_{0} \\
{[\mathrm{mag}]}\end{array}$ & $\begin{array}{c}\log T_{\text {eff }} \\
{[\mathrm{dex}]}\end{array}$ & $\begin{array}{c}\log g \\
{[\mathrm{dex}]}\end{array}$ & $\begin{array}{c}v \sin i \\
{\left[\mathrm{~km} \mathrm{~s}^{-1}\right]}\end{array}$ & $\begin{array}{c}{[Z]} \\
{[\mathrm{dex}]}\end{array}$ & $\begin{array}{c}M_{V} \\
{[\mathrm{mag}]}\end{array}$ & $\begin{array}{c}M_{B} \\
{[\mathrm{mag}]}\end{array}$ & $\begin{array}{c}\log L_{*} / L_{\odot} \\
{[\mathrm{dex}]}\end{array}$ & $\begin{array}{c}\log P \\
{[\mathrm{~d}]}\end{array}$ \\
\hline 185139 & 0.157 & $3.869(2)$ & $3.80(7)$ & - & +0.29 & $1.38(51)$ & 1.29 & $1.39(20)$ & -1.19 & 0.018 \\
186786 & 0.181 & $3.856(2)$ & $3.86(8)$ & - & +0.19 & $2.09(3)$ & 2.00 & $1.10(1)$ & -1.10 & 0.027 \\
188520 & 0.123 & $3.885(7)$ & $4.05(15)$ & - & +0.03 & $2.19(19)$ & 2.08 & $1.07(8)$ & -1.26 & 0.025 \\
199124 & 0.167 & $3.859(3)$ & $3.74(8)$ & 150 & -0.12 & $1.89(13)$ & 1.80 & $1.18(5)$ & -1.00 & 0.028 \\
199908 & 0.192 & $3.848(4)$ & $3.42(7)$ & 60 & +0.27 & $1.23(21)$ & 1.13 & $1.45(8)$ & -1.10 & 0.013 \\
206553 & 0.171 & $3.859(4)$ & $3.70(15)$ & - & +0.20 & $1.46(2)$ & 1.37 & $1.35(1)$ & -1.20 & 0.016 \\
208435 & 0.198 & $3.844(5)$ & $3.26(8)$ & - & +0.36 & $0.67(43)$ & 0.56 & $1.68(17)$ & -0.83 & 0.017 \\
211336 & 0.170 & $3.862(3)$ & $3.90(7)$ & 90 & +0.17 & $2.12(2)$ & 2.03 & $1.09(1)$ & -1.39 & 0.015 \\
214441 & 0.205 & $3.847(4)$ & $3.55(11)$ & - & +0.45 & $1.28(59)$ & 1.18 & $1.43(23)$ & -0.90 & 0.024 \\
215874 & 0.163 & $3.863(4)$ & $3.48(5)$ & 100 & +0.23 & $0.88(20)$ & 0.79 & $1.58(8)$ & -1.06 & 0.015 \\
217236 & 0.152 & $3.868(4)$ & $3.52(8)$ & 100 & +0.22 & $0.51(59)$ & 0.42 & $1.73(24)$ & -0.90 & 0.020 \\
219891 & 0.076 & $3.902(8)$ & $3.77(15)$ & 165 & -0.02 & $0.80(6)$ & 0.67 & $1.63(3)$ & -1.00 & 0.025 \\
220061 & 0.100 & $3.882(17)$ & $3.51(2)$ & 140 & +0.04 & $0.95(14)$ & 0.85 & $1.56(6)$ & -1.27 & 0.010 \\
223781 & 0.098 & $3.897(4)$ & $3.92(12)$ & 165 & -0.20 & $1.47(8)$ & 1.34 & $1.37(3)$ & -1.22 & 0.021 \\
\hline
\end{tabular}

For the remaining 20 stars only photometrically calibrated absolute magnitudes are available. We then calculated luminosities $\left(\log L_{*} / L_{\odot}\right)$ using the absolute bolometric magnitude of the Sun $M_{\mathrm{Bol}}(\odot)=4.75 \mathrm{mag}$ (Cayrel de Strobel 1996) and bolometric corrections taken from Drilling \& Landolt (2000).

For HD 84948B we have used the astrophysical parameters listed by Iliev et al. (2002; Table 1). This is an evolved spectroscopic binary system which contains two similar $\lambda$ Bootis components; Iliev et al. (2002) have taken the binary nature into account.

Individual abundances and projected rotational velocities for members of the $\lambda$ Bootis group were published by Uesugi \& Fukuda (1982), Venn \& Lambert (1990), Stürenburg (1993), Abt \& Morrell (1995), Holweger \& Rentzsch-Holm (1995), Chernyshova et al. (1998), Heiter et al. (1998), Paunzen et al. (1999a,b), Kamp et al. (2001), Solano et al. (2001), Heiter (2002) and Andrievsky et al. (2002). The individual values were weighted (if possible) with the errors listed in the references and averaged.

The published abundances do not allow an investigation of the correlation of individual abundances of different elements (which have different diffusion properties) with the pulsational period. It is well known that the typical abundance pattern of $\lambda$ Bootis stars is characterized by moderate to strong underabundances of elements heavier than $\mathrm{C}, \mathrm{N}, \mathrm{O}$ and $\mathrm{S}$. To get an overall estimate of the (surface) abundance we have applied the following method:

- A weighted mean for $\mathrm{Mg}, \mathrm{Ca}, \mathrm{Sc}, \mathrm{Ti}, \mathrm{Cr}$ and $\mathrm{Fe}$ was calculated and taken as a measurement of $[Z]$. This should minimize measurement errors for individual elements;

- We determined $\Delta m_{2}$ from the Geneva 7-colour as well as $\Delta m_{1}$ from the Strömgren $u v b y \beta$ photometric system (for the definition of these parameters see Golay 1974);

- Then we correlated $\Delta m_{2}$ or $\Delta m_{1}$ with [Z] for stars without published individual element abundances.

The third step is only valid for effective temperatures cooler than $8500 \mathrm{~K}$; otherwise the metallicity indices are no longer

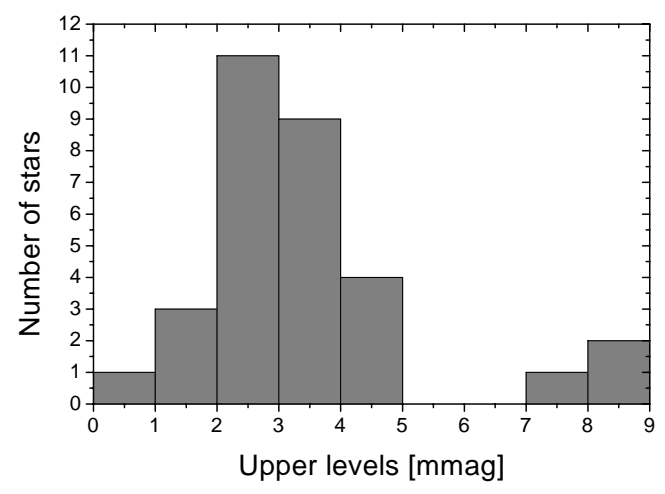

Fig. 3. The histogram of our upper levels for non-variability. See text for details.

sensitive. This method was applied to nine pulsating $\lambda$ Bootis type objects: HD 6870, HD 30422, HD 42503, HD 102541, HD 109738, HD 110377, HD 153747, HD 168947 and HD 213669.

\section{Results}

Besides two objects (HD 125889 and HD 184779), all members of the $\lambda$ Bootis group were photometrically investigated. All previously published results were taken from Paunzen et al. $(1997,1998)$ as well as from the references quoted in Sect. 3. Of these 65 stars, 32 are presumed to be constant whereas 33 are pulsating. The upper limits for non-variability, which are below 5 mmag for all but three stars (HD 31925, HD 91130 and HD 294253), are shown in Fig. 3.

In order to investigate the pulsational characteristics of the $\lambda$ Bootis stars as a group, we compare them with those of "normal" $\delta$ Scuti variables. The next subsection describes the compilation of the latter sample. 

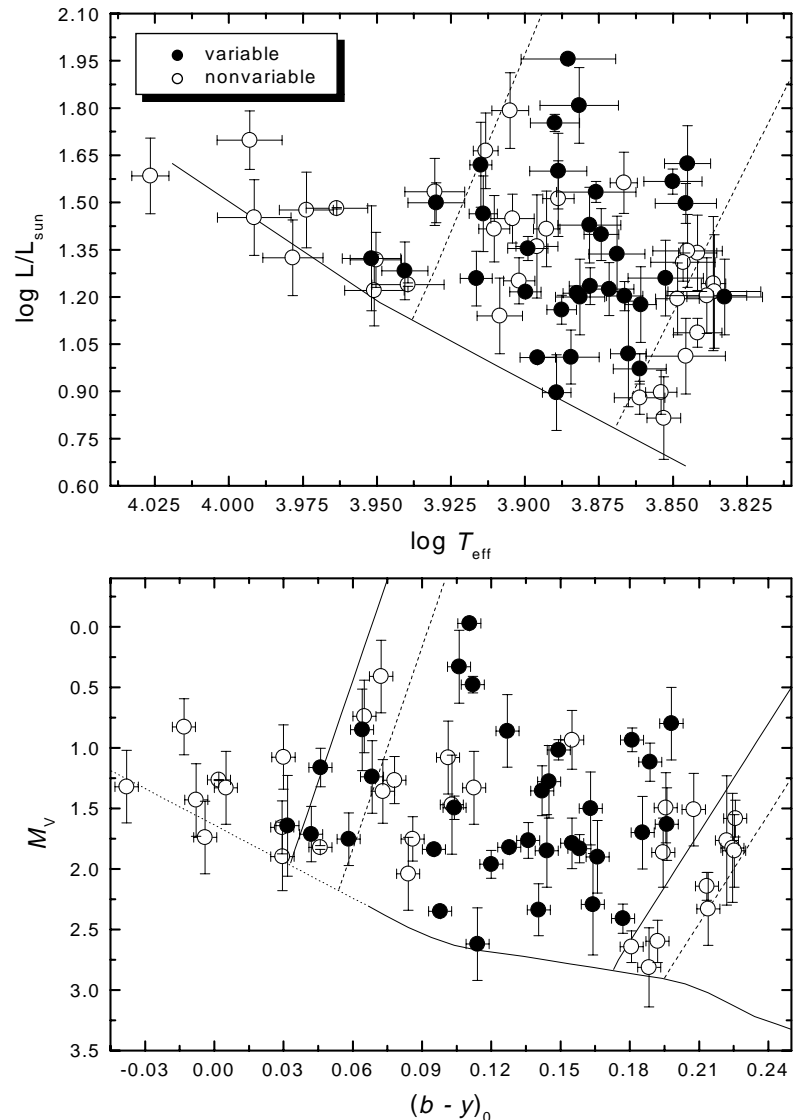

Fig. 4. The $\log L_{*} / L_{\odot}$ versus $\log T_{\text {eff }}$ (upper panel) and $M_{V}$ versus $(b-y)_{0}$ (lower panel) diagrams for the non-variable (open circles) and pulsating (filled circles) $\lambda$ Bootis stars. The Zero Age Main Sequences are taken from Crawford (1979) and Claret (1995). The borders of the classical instability strip (dotted lines) are taken from Breger (1995). The observed borders from our sample are indicated as filled lines in the lower panel.

\subsection{A sample of $\delta$ Scuti stars}

As a basis we have used the catalogue of Rodriguez et al. (2000). From this sample, stars have been rejected following these criteria:

- Classification as Am, Ap, $\delta$ Delphini and SX Phoenicis objects;

- $v \sin i<45 \mathrm{~km} \mathrm{~s}^{-1}$ (if available);

- $\sigma(\pi) / \pi>0.175$

- $\log P<-1.7$ and $\log P>-0.8$;

- Amplitude $>0.08$ mag (if no $v \sin i$ available);

- without Johnson and Geneva photometry;

- $E(b-y)>0.05 \mathrm{mag}$.

Such a choice is based on the characteristics of our $\lambda$ Bootis type sample and is hoped to guarantee a comparable sample of $\delta$ Scuti type stars. In total, 69 objects remain in the sample. The basic parameters, etc. were derived in exactly the same way as for the $\lambda$ Bootis type objects, as described in Sect. 3. Table 5 lists all calibrated parameters together with the periods given by Rodriguez et al. (2000).
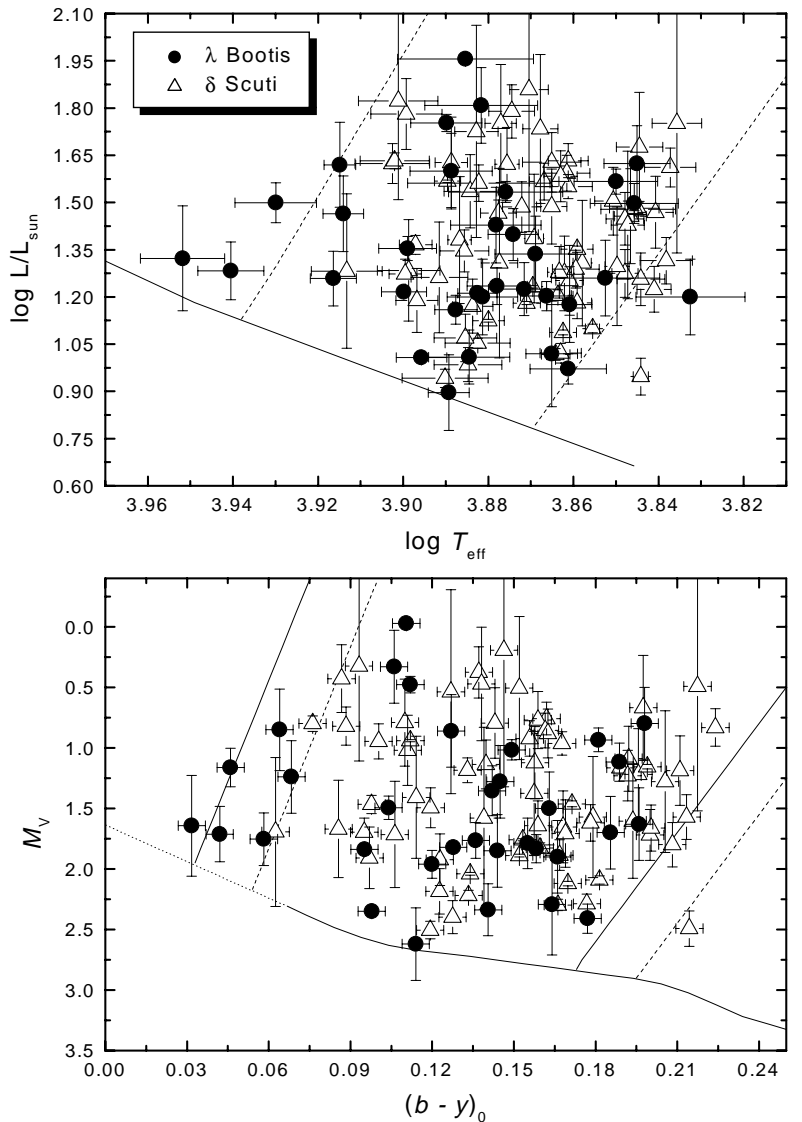

Fig. 5. The $\log L_{*} / L_{\odot}$ versus $\log T_{\text {eff }}$ (upper panel) and $M_{V}$ versus $(b-y)_{0}$ (lower panel) diagrams for pulsating $\lambda$ Bootis (filled circles) and selected $\delta$ Scuti (open triangles) stars. The location of both samples are comparable justifying our selection criteria of the $\delta$ Scuti type stars. The lines are the same as in Fig. 4.

\subsection{Hertzsprung-Russell-diagram and the pulsational characteristics}

First of all, we have investigated the location of all $\lambda$ Bootis stars within the $\log L_{*} / L_{\odot}$ versus $\log T_{\text {eff }}$ and $M_{V}$ versus $(b-y)_{0}$ diagrams (Fig. 4). The borders of the classical instability strip are taken from Breger (1995). There are several conclusions from this figure:

- The published hot and cool borders of the $\delta$ Scuti instability strip within the $M_{V}$ versus $(b-y)_{0}$ diagram do not coincide with the observed ones for the $\lambda$ Bootis stars. The latter are bluer at the Zero Age Main Sequence (ZAMS hereafter) by about 25 mmag. However, the borders are in accordance with the observations within the $\log L_{*} / L_{\odot}$ versus $\log T_{\text {eff }}$ diagram;

- Taking the average of variable to non-variable objects within the classical instability strip for both diagrams then we derive a value of at least $70 \%$ pulsating objects.

Figure 5 shows the same diagrams for this sample (filled circles) together with those of the selected $\delta$ Scuti stars (open triangles). Besides one object (HD 57167), the cool borders are in excellent agreement with the observations. However, there are four hot $\lambda$ Bootis type pulsators: HD 120500, HD 125162, 


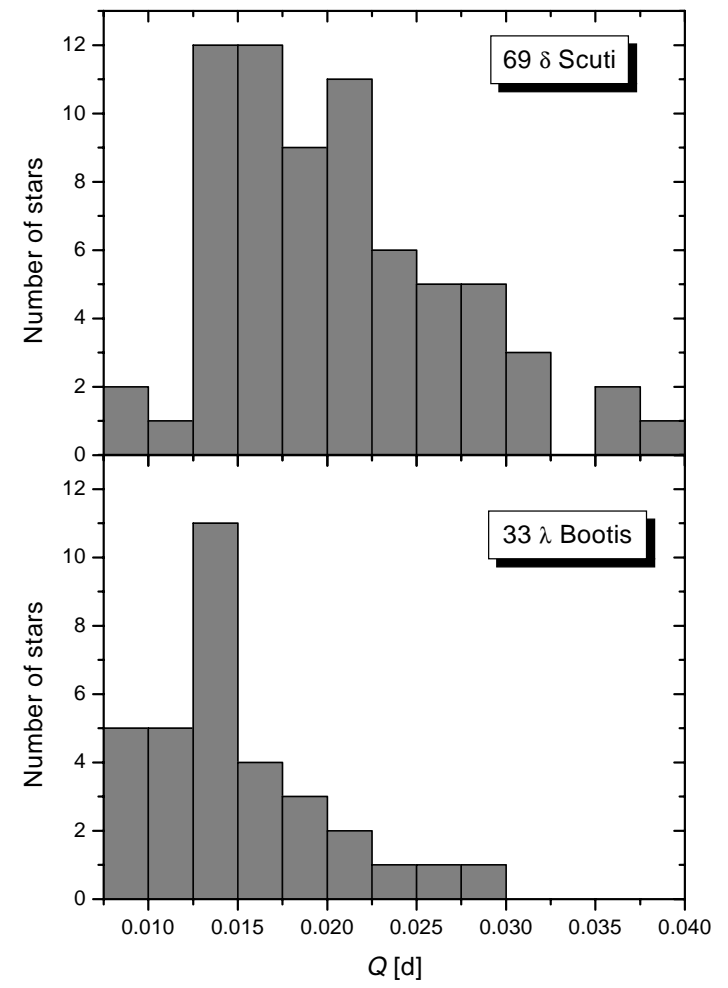

Fig. 6. The histograms of the pulsational constant $Q$ for the selected $\delta$ Scuti (upper panel) and $\lambda$ Bootis (lower panel) stars.

HD 183324 and HD 221756. The reason for these shifts is not yet clear. We are able to exclude measurements errors (mean values from several references were used) and the effects of rotation (all stars have moderate $v \sin i$ values). In addition, taking the unreddened colours, all four stars are still outside the hot border. Therefore it is somewhat surprising that only one object (HD 183324) lies significantly outside the borders within the $\log L_{*} / L_{\odot}$ versus $\log T_{\text {eff }}$ diagram.

As a next step towards analyzing the pulsational characteristics we have calculated the pulsation constants given by

$\log Q=-6.456+0.5 \log g+0.1 M_{B}+\log T_{\text {eff }}+\log P$.

The resulting $Q$-values are listed in Table 4 and in Table 5 for our program $\lambda$ Bootis stars and for the comparison sample of $\delta$ Scuti stars, respectively. For the $\lambda$ Bootis group, the $Q$-values range from 0.038 to 0.033 for the fundamental radial modes and decrease to about 0.012 for the fifth radial overtone (Stellingwerf 1979; Fitch 1981). Figure 6 (lower panel) shows a histogram of the $Q$-values for the pulsating program stars. It seems that only a few stars, if any, pulsate in the fundamental mode, but there is a high percentage with $Q<0.020 \mathrm{~d}$ (high overtone modes).

The distribution of the $Q$-values for the $\lambda$ Bootis type stars is different from that of the $\delta$ Scuti type sample (Fig. 6, upper panel) at a $99.9 \%$ level (derived from a $t$-test).

We also noticed four pulsators (HD 15165, HD 42503, HD 111604 and HD 142994) that have considerably longer periods ( $\log P>-0.94$ corresponding to $P<8.7 \mathrm{~d}^{-1}$ ) than the rest of our sample. They do, however, show a similar behaviour to the remaining group members.
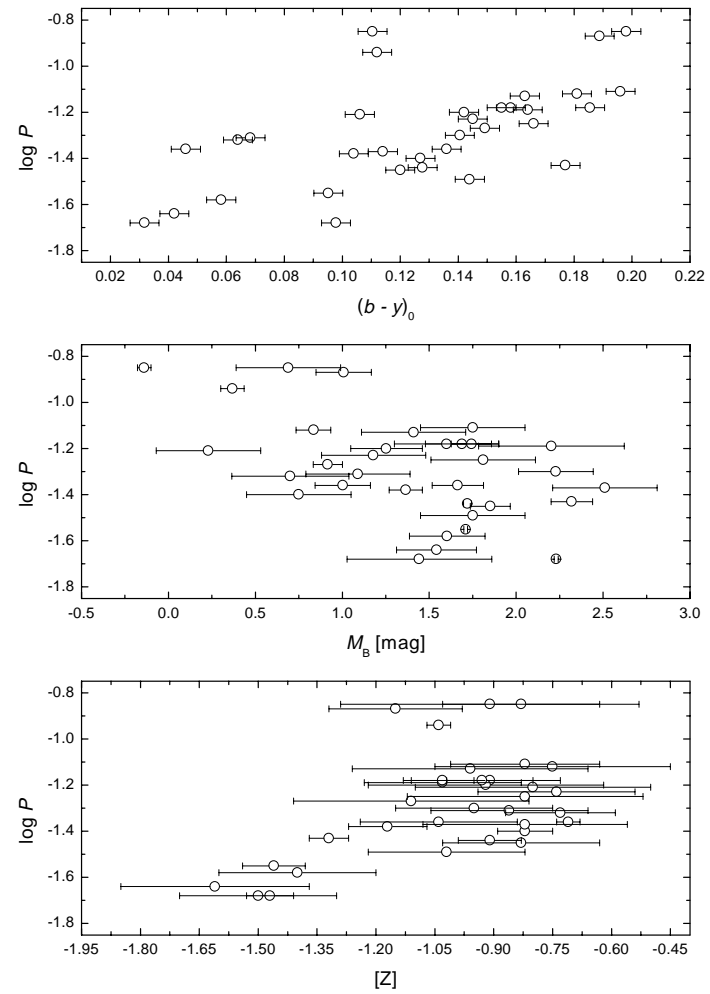

Fig. 7. Correlation of $(b-y)_{0}$, the absolute magnitude and metallicity with $\log P$ for all $\lambda$ Bootis type stars.

\subsection{The Period-Luminosity-Colour-Metallicity (PLCZ) relation}

A Period-Luminosity-Metallicity relation was found for Population II type variables such as RR Lyrae and SX Phoenicis stars as well as Cepheids (Nemec et al. 1994). These objects pulsate in the radial fundamental, first and second overtone modes. The dependence of the pulsational period on the metallicity is purely evolutionary, i.e. older objects exhibit a lower overall abundance and a different pulsational period. The Period-Luminosity-Metallicity relation serves as a distance indicator widely used for extragalactic objects.

The situation for $\lambda$ Bootis stars is very different. All evidence indicates that we find only peculiar surface abundances whereas the overall abundance of the stars is solar, i.e. these stars are true Population I objects. The conclusion that $\lambda$ Bootis stars are true Population I objects is based on their galactic space motions (Faraggiana \& Bonifacio 1999) combined with their location in the Hertzsprung-Russell-diagram (Fig. 4). With the exception of the SX Phe stars, Population II type objects are located at much higher absolute magnitudes and thus luminosities than found for the $\lambda$ Bootis group. However, the space motions of SX Phe stars are inconsistent with Population I, which facilitates an easy separation from $\lambda$ Bootis stars.

To examine the presence of a PLCZ relation, the following basic approach was chosen:

$\log P=a+b(b-y)_{0}+c\left(M_{B}\right)+d[Z]$.

The $[Z]$-values range from $-0.71 \mathrm{dex}$ to $-1.61 \mathrm{dex}$ for the $\lambda$ Bootis type sample whereas the $\delta$ Scuti type stars have 
Table 6. Nonvariable $\lambda$ Bootis stars, an asterisk denotes stars without an accurate HIPPARCOS parallax; $\sigma(b-y)= \pm 0.005$ mag. In parenthesis are the errors in the final digits of the corresponding quantity.

\begin{tabular}{lllll}
\hline \hline \multicolumn{1}{c}{ HD } & $\begin{array}{c}(b-y)_{0} \\
{[\mathrm{mag}]}\end{array}$ & $\log T_{\text {eff }}$ & $\begin{array}{c}M_{V} \\
{[\mathrm{mag}]}\end{array}$ & $\log L_{*} / L_{\odot}$ \\
\hline 319 & +0.078 & $3.904(7)$ & $1.27(19)$ & $1.45(8)$ \\
7908 & +0.192 & $3.854(5)$ & $2.60(18)$ & $0.90(7)$ \\
$23392^{*}$ & -0.008 & $3.991(12)$ & $1.43(30)$ & $1.45(12)$ \\
24472 & +0.213 & $3.842(8)$ & $2.14(11)$ & $1.09(5)$ \\
31295 & +0.029 & $3.950(9)$ & $1.66(22)$ & $1.32(9)$ \\
$36726^{*}$ & -0.004 & $3.978(10)$ & $1.74(30)$ & $1.32(12)$ \\
$54272^{*}$ & +0.214 & $3.846(13)$ & $2.33(30)$ & $1.01(12)$ \\
74873 & +0.046 & $3.940(12)$ & $1.82(1)$ & $1.24(1)$ \\
$81290^{*}$ & +0.225 & $3.839(13)$ & $1.85(30)$ & $1.20(12)$ \\
83277 & +0.196 & $3.845(12)$ & $1.49(29)$ & $1.35(12)$ \\
84123 & +0.226 & $3.847(11)$ & $1.58(15)$ & $1.31(6)$ \\
$90821^{*}$ & +0.065 & $3.913(4)$ & $0.74(30)$ & $1.66(12)$ \\
91130 & +0.073 & $3.910(5)$ & $1.36(26)$ & $1.42(11)$ \\
$101108^{*}$ & +0.113 & $3.893(4)$ & $1.33(30)$ & $1.42(12)$ \\
106223 & +0.225 & $3.836(16)$ & $1.83(45)$ & $1.22(18)$ \\
107233 & +0.181 & $3.861(9)$ & $2.64(13)$ & $0.88(5)$ \\
110411 & +0.029 & $3.951(10)$ & $1.90(28)$ & $1.22(11)$ \\
111005 & +0.222 & $3.836(4)$ & $1.76(53)$ & $1.24(21)$ \\
130767 & +0.002 & $3.964(10)$ & $1.27(2)$ & $1.48(1)$ \\
$149130^{*}$ & +0.208 & $3.842(6)$ & $1.51(30)$ & $1.34(12)$ \\
154153 & +0.194 & $3.848(7)$ & $1.86(29)$ & $1.19(11)$ \\
156954 & +0.188 & $3.853(6)$ & $2.81(33)$ & $0.82(13)$ \\
170680 & -0.013 & $3.993(11)$ & $0.83(23)$ & $1.70(9)$ \\
175445 & +0.030 & $3.930(10)$ & $1.08(27)$ & $1.53(11)$ \\
$193256^{*}$ & +0.101 & $3.889(5)$ & $1.08(30)$ & $1.51(12)$ \\
$193281^{*}$ & +0.072 & $3.905(6)$ & $0.41(30)$ & $1.79(12)$ \\
198160 & +0.103 & $3.896(7)$ & $1.47(41)$ & $1.36(16)$ \\
204041 & +0.086 & $3.902(5)$ & $1.75(18)$ & $1.25(7)$ \\
216847 & +0.155 & $3.867(5)$ & $0.93(24)$ & $1.56(10)$ \\
$261904^{*}$ & +0.005 & $3.974(9)$ & $1.33(30)$ & $1.48(12)$ \\
$290492^{*}$ & +0.084 & $3.908(8)$ & $2.04(30)$ & $1.14(12)$ \\
$294253^{*}$ & -0.038 & $4.027(6)$ & $1.32(30)$ & $1.58(12)$ \\
\hline & & & &
\end{tabular}

values from -0.36 dex to +0.78 dex compared to the Sun. The coefficients for the PLCZ relation were determined simultaneously, applying a multiregression analysis (Christensen 1996). This takes into account the individual errors of the bolometric absolute magnitude and metallicity as weights, whereas the errors for the period and colour were assumed to be constant for all stars. The solution was determined using a least-squares fit and a maximum-likelihood method. Both give consistent results, as summarized in Table 8. Figure 7 shows the individual correlations. The correlations of the bolometric magnitude and color with the pulsational period are compatible with those found for the $\delta$ Scuti stars.

To investigate whether the $[Z]$ term is indeed significant, a plot $\left[\log P-2.86(b-y)_{0}+0.195\left(M_{B}\right)\right]$ versus $[Z]$ was drawn (Fig. 8). The coefficients for $(b-y)_{0}$ and $M_{B}$ are the mean values from Table 8 and are consistent within the errors for both the $\delta$ Scuti and $\lambda$ Bootis samples. Figure 8 shows that both samples exhibit a trend with $[Z]$ (with an offset of about 1 dex). Whereas
Table 7. Comparison of effective temperatures and surface gravities for $\lambda$ Bootis stars from Heiter et al. (2002; Table 1; columns "Literature") and this work; $\Delta T_{\text {eff }}=T_{\text {eff }}($ Lit. $)-T_{\text {eff }}(\mathrm{TW})=$ $+72(210) \mathrm{K} ; \Delta \log g=\log g$ (Lit.) $-\log g(\mathrm{TW})=+0.07(24)$ dex.

\begin{tabular}{|c|c|c|c|c|}
\hline \multirow[b]{2}{*}{ HD } & \multicolumn{2}{|c|}{ Literature } & \multicolumn{2}{|c|}{ This work } \\
\hline & $\begin{array}{c}T_{\text {eff }} \\
\pm 200 \mathrm{~K}\end{array}$ & $\begin{array}{c}\log g \\
\pm 0.3 \mathrm{dex}\end{array}$ & $T_{\text {eff }}$ & $\log g$ \\
\hline 319 & 8100 & 3.8 & $8020(135)$ & $3.74(8)$ \\
\hline 11413 & 7900 & 3.8 & $7925(124)$ & $3.91(21)$ \\
\hline 15165 & 7200 & 3.7 & $7010(167)$ & $3.23(10)$ \\
\hline 31295 & 8800 & 4.2 & $8920(177)$ & $4.20(1)$ \\
\hline 74873 & 8900 & 4.6 & $8700(245)$ & $4.21(11)$ \\
\hline 75654 & 7250 & 3.8 & $7350(104)$ & $3.77(11)$ \\
\hline 81290 & 6780 & 3.5 & $6895(214)$ & $3.82(28)$ \\
\hline 84123 & 6800 & 3.5 & $7025(145)$ & $3.73(17)$ \\
\hline 101108 & 7900 & 4.1 & $7810(90)$ & $3.90(18)$ \\
\hline 105759 & 8000 & 4.0 & $7485(102)$ & $3.65(10)$ \\
\hline 106223 & 7000 & 4.3 & $6855(247)$ & $3.49(18)$ \\
\hline 107233 & 7000 & 3.8 & $7265(143)$ & $4.03(10)$ \\
\hline 109738 & 7575 & 3.9 & $7610(145)$ & $3.90(13)$ \\
\hline 110411 & 9100 & 4.5 & $8930(206)$ & $4.14(14)$ \\
\hline 111005 & 7410 & 3.8 & $6860(66)$ & $3.72(10)$ \\
\hline 125162 & 8650 & 4.0 & $8720(156)$ & $4.07(9)$ \\
\hline 142703 & 7100 & 3.9 & $7265(150)$ & $3.93(12)$ \\
\hline 156954 & 6990 & 4.1 & 7130(93) & $4.04(13)$ \\
\hline 168740 & 7700 & 3.7 & $7630(81)$ & $3.88(14)$ \\
\hline 170680 & 10000 & 4.1 & $9840(248)$ & $4.15(6)$ \\
\hline 183324 & 9300 & 4.3 & $8950(204)$ & $4.13(4)$ \\
\hline 192640 & 7960 & 4.0 & 7940(96) & $3.95(18)$ \\
\hline 193256 & 7800 & 3.7 & 7740(94) & $3.69(17)$ \\
\hline 193281 & 8070 & 3.6 & $8035(115)$ & $3.54(4)$ \\
\hline 198160 & 7900 & 4.0 & 7870(129) & 3.99(9) \\
\hline 204041 & 8100 & 4.1 & 7980(97) & $3.97(8)$ \\
\hline 210111 & 7530 & 3.8 & $7550(123)$ & $3.84(15)$ \\
\hline 221756 & 9010 & 4.0 & $8510(188)$ & $3.90(3)$ \\
\hline
\end{tabular}

Table 8. Estimates for $\log P=a+b(b-y)_{0}+c\left(M_{B}\right)+d[Z]$ for all pulsating program stars (left upper column), for stars with $[Z]>-1.3$ excluding HD 30422, HD 35242, HD 125162, HD 142703, HD 183324 and HD 192640 (right upper column) as well as for the selected $\delta$ Scuti stars (lower column); $F$ denotes the significance level of the test for a zero hypothesis; in brackets are the standard errors of the estimates.

\begin{tabular}{lll|ll}
\hline \hline coeff. & value & $\begin{array}{c}F \\
{[\%]}\end{array}$ & value & $\begin{array}{c}F \\
{[\%]}\end{array}$ \\
\hline$\lambda$ Bootis & & \multicolumn{1}{c}{ [\% } & \\
$a$ & $-1.25(9)$ & $<0.01$ & $-1.41(9)$ & $<0.01$ \\
$b(b-y)_{0}$ & $+3.01(41)$ & $<0.01$ & $+2.71(46)$ & $<0.01$ \\
$c\left(M_{B}\right)$ & $-0.20(3)$ & $<0.01$ & $-0.19(3)$ & $<0.01$ \\
$d[Z]$ & $+0.14(6)$ & 13.20 & $-0.06(17)$ & 74.49 \\
\hline$\delta$ Scuti & \multicolumn{5}{|c}{} \\
$a$ & $-1.17(6)$ & $<0.01$ & & \\
$b(b-y)_{0}$ & $+2.71(43)$ & $<0.01$ & & \\
$c\left(M_{B}\right)$ & $-0.23(3)$ & $<0.01$ & \\
$d[Z]$ & $-0.28(7)$ & $<0.01$ & & \\
\hline
\end{tabular}




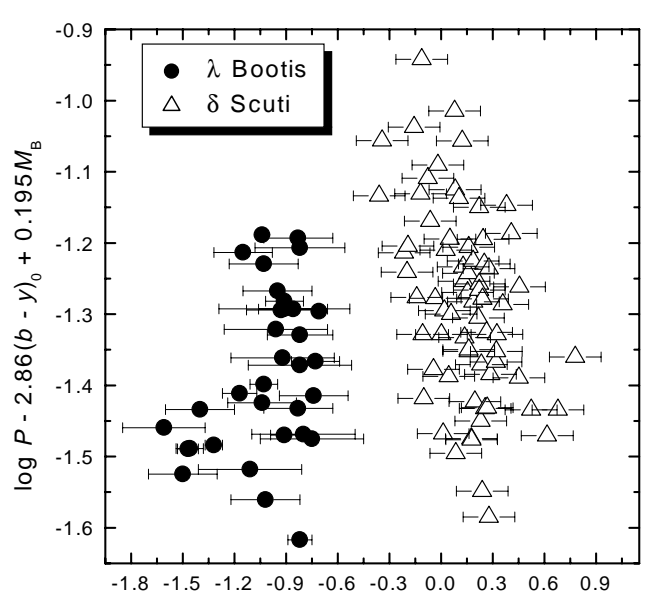

[Z]

Fig. 8. Correlation of $[Z]$ for the $\lambda$ Bootis (filled circles) and selected $\delta$ Scuti type (open triangles) stars.

the $[Z]$ term is statistically significant for the selected sample of $\delta$ Scuti stars, it is caused by only six $\lambda$ Bootis stars with strong underabundances (HD 30422, HD 35242, HD 125162 , HD 142703, HD 183324 and HD 192640) and vanishes after excluding them. We have also tested the sample for possible correlations of the $[Z]$ term by excluding other data points. We find no other selection criteria of objects by means of a physical explanation, only "suitable" discarding would yield a clear correlation. This implies that the peculiar abundances do not affect the pulsational period for the group of $\lambda$ Bootis type stars. However, we find within the errors no difference of the PLC relation for the $\lambda$ Bootis and $\delta$ Scuti type stars.

\section{Conclusions}

We have investigated the pulsational characteristics of a group of $\lambda$ Bootis stars and compared it to a sample of $\delta$ Scuti pulsators. The latter was chosen such that it matches our program stars within the global astrophysical parameters. The following properties of the $\lambda$ Bootis stars are different from those of the $\delta$ Scuti pulsators:

- At least $70 \%$ of all $\lambda$ Bootis types stars inside the classical instability strip pulsate.

- Only a maximum of two stars pulsate in the fundamental mode but there is a high percentage with $Q<0.020 \mathrm{~d}$ (high overtone modes).

- The instability strip of the $\lambda$ Bootis stars at the ZAMS is 25 mmag bluer in $(b-y)_{0}$ than that of the $\delta$ Scuti stars.

We find no clear evidence for a significant term for a $[Z]$ correlation with the period, luminosity and colour but the PLC relation is within the errors identical with that of the $\delta$ Scuti type stars. We note that for all but one of the investigated pulsators, high-degree nonradial modes were detected spectroscopically (Bohlender et al. 1999), which represents excellent agreement with our work. The spectral variability of the $\lambda$ Bootis stars is very similar to that seen in rapidly rotating $\delta$ Scuti stars (Kennelly et al. 1992).
Acknowledgements. This work benefitted from the Fonds zur Förderung der wissenschaftlichen Forschung, project P14984. ERC would like to thank D. Romero, E. Colmenero and S. Potter for their support. Use was made of the SIMBAD database, operated at CDS, Strasbourg, France and the GCPD database, operated at the Institute of Astronomy of the University of Lausanne. We are also indebted to the committees of the SAAO, Siding Spring and Fairborn Observatory who granted observing time.

\section{References}

Abt, H. A. 1984, in The MK Process and Stellar Classification, ed. R. F. Garrison (David Dunlap Observatory, Toronto), 340

Abt, H. A., \& Morrell, N. I. 1995, ApJS, 99, 135

Andrievsky, S. M. 1997, A\&A, 321, 838

Andrievsky, S. M., Chernyshova, I. V., Korotin, S. A., et al. 2002, A\&A, in preparation

Antonello, E., \& Mantegazza, L. 1982, A\&AS, 49, 709

Arenou, F., Grenon, M., \& Gómez, A. 1992, A\&A, 258, 104

Balona, L. A. 1977, Mem. R. Astr. Soc., 84, 101

Bartolini, C., Dapergolas, A., Piccioni, A., \& Voli, M. 1980a, IBVS, 1753

Bartolini, C., Dapergolas, A., Piccioni, A., \& Voli, M. 1980b, IBVS, 1757

Bohlender, D. A., Gonzalez, J. F., \& Matthews, J. M. 1999, A\&A, 350, 553

Breger, M. 1979, PASP, 91, 5

Breger, M. 1995, PASPC, 76, 596

Cayrel de Strobel, G. 1996, A\&AR, 7, 243

Charbonneau, P. 1993, ApJ, 405, 720

Chen, B., Vergely, J. L., Valette, B., \& Carraro, G. 1998, A\&A, 336, 137

Chernyshova, I. V., Andrievsky, S. M., Kovtyukh, V. V., \& Mkrtichian, D. E. 1998, Contrib. Astron. Obs. Skalnaté Pleso, 27(3), 332

Christensen, R. 1996, Analysis of Variance, Design and Regression, Applied statistical methods (Chapman \& Hall, London), 391

Claret, A. 1995, A\&AS, 109, 441

Crawford, D. L. 1979, AJ, 84, 1858

Deeming, T. J. 1975, Ap\&SS, 36, 137

Desikachary, K., \& McInally, C. J. 1979, MNRAS, 188, 67

Drilling, J. S., \& Landolt, A. U. 2000, in Allen's Astrophysical Quantities, 4th edition, ed. A. N. Cox (Springer Verlag), 388

ESA (European Space Agency) 1997, The Hipparcos and Tycho Catalogues (ESA Publications, Noordwijk)

Faraggiana, R., \& Bonifacio, P. 1999, A\&A, 349, 521

Fitch, W. S. 1981, ApJ, 249, 218

Gerbaldi, M., Faraggiana, R., Burnage, R., et al. 1999, A\&AS, 137, 273

Gies, D. R., \& Percy, J. R. 1977, AJ, 82, 166

Golay, M. 1974, Introduction to astronomical photometry (D. Reidel Publishing Co., Dordrecht)

Gray, R. O. 1988, AJ, 95, 220

Gray, R. O., \& Corbally, C. J. 1993, AJ, 106, 632

Hakkila, J., Myers, J. M., Stidham, B. J., \& Hartmann, D. H. 1997, AJ, 114, 2043

Handler, G. 1999, IBVS, 4817

Handler, G. 2002, in Observational Aspects of Pulsating B- and A Stars, ed. C. Sterken, \& D. W. Kurtz (San Francisco: Astronomical Society of the Pacific), ASP Conf. Proc., 256, 113

Handler, G., Gray, R. O., \& Shobbrook, R. R. 2000, IBVS, 4876

Heiter, U. 2002, A\&A, 381, 959

Heiter, U., Kupka, F., Paunzen, E., Weiss, W. W., \& Gelbmann, M. 1998, A\&A, 335, 1009 
Heiter, U., Weiss, W. W., \& Paunzen, E. 2002, A\&A, 381, 971

Hilditch, R. W., Hill, G., \& Barnes, J. V. 1983, MNRAS, 204, 241

Holweger, H., \& Rentzsch-Holm, I. 1995, A\&A, 303, 819

Iliev, I. Kh., Paunzen, E., Barzova, I. S., et al. 2001, IBVS, 5199

Iliev, I. Kh., Paunzen, E., Barzova, I. S., et al. 2002, A\&A, 381, 914

Kamp, I., Iliev, I. Kh., Paunzen, E., et al. 2001, A\&A, 375, 899

Kennelly, E. J., Walker, G. A. H., \& Merryfield, W. J. 1992, ApJ, 400, L71

Kjeldsen, H., \& Frandsen, S. 1992, PASP, 104, 413

Kobi, D., \& North, P. 1990, A\&AS, 85, 999

Koen, C. 1992, MNRAS, 256, 65

Künzli, M., North, P., Kurucz, R. L., \& Nicolet, B. 1997, A\&AS, 122, 51

Kupka, F., Piskunov, N. E., Ryabchikova, T. A., Stempels, H. C., \& Weiss, W. W. 1999, A\&AS, 138, 1

Kurtz, D. W. 2000, in Delta Scuti and Related Stars, ed. M. Breger, \& M. H. Montgomery, ASP Conf. Ser., 210, 287

Kusakin, A. V., \& Mkrtichian, D. E. 1996, IBVS, 4314

Liu, Y. Y., Jiang, S. Y., Michel, E., et al. 1996, A\&AS, 120, 179

Martinez, P., Koen, C., Handler, G., \& Paunzen, E. 1998, MNRAS, 301, 1099

Michaud, G., \& Charland, Y. 1986, ApJ, 311, 326

Mkrtichian, D. E., Kusakin, A. V., Koval, V. A., et al. 2000, in The Impact of Large-Scale Surveys on Pulsating Star Research, ed. I. Szabados, \& D. Kurtz, ASP Conf. Ser., 203, 494

Moon, T. T., \& Dworetsky, M. M. 1985, MNRAS, 217, 305

Napiwotzki, R., Schönberner, D., \& Wenske V. 1993, A\&A, 268, 653

Nemec, J. M., Nemec, A. F. L., \& Lutz, T. E. 1994, AJ, 108, 222
Oudmaijer, R. D., Groenewegen, M. A. T., \& Schrijver, H. 1998, MNRAS, 294, L41

Paunzen, E. 2001, A\&A, 373, 633

Paunzen, E., \& Handler, G. 1996, IBVS, 4318

Paunzen, E., \& Gray, R. O. 1997, A\&AS, 126, 407

Paunzen, E., Kuschnig, R., Handler, G., Gelbmann, M., \& Weiss, W. W. 1997, A\&AS, 124, 23

Paunzen, E., Weiss, W. W., Kuschnig, R., et al. 1998, A\&A, 335, 533

Paunzen, E., Kamp, I., \& Iliev, I. Kh. 1999a, A\&A, 351, 981

Paunzen, E., Andrievsky, S. M., Chernyshova, I. V., et al. 1999b, A\&A, 351, 981

Rodriguez, E., Lopez-Gonzalez, M. J., \& Lopez de Coca, P. 2000, A\&AS, 144, 469

Sandage, A. 1972, ApJ, 178, 1

Solano, E., Paunzen, E., Pintado, O. I., \& Varela, J. 2001, A\&A, 374, 957

Stellingwerf, R. F. 1978, ApJ, 224, 953

Stellingwerf, R. F. 1979, ApJ, 227, 935

Strömgren, B. 1966, ARA\&A, 4, 433

Stürenburg, S. 1993, A\&A, 277, 139

Turcotte, S., \& Charbonneau, P. 1993, ApJ, 413, 376

Turcotte, S., Richer, J., Michaud, G., \& Christensen-Dalsgaard, J. 2000, A\&A, 360, 603

Uesugi, A., \& Fukuda, I. 1982, Revised Catalogue of Stellar Rotational Velocities, Department of Astronomy, Kyoto Univ., Kyoto

Venn, K. A., \& Lambert, D. L. 1990, ApJ, 363, 234

Waelkens, C., \& Rufener, F. 1983, Hvar Obs. Bull., 7, 301 